\title{
Erratum to: On the viscosity solutions to Trudinger's equation
}

Tilak Bhattacharya and Leonardo Marazzi

Erratum to: Nonlinear Differ. Equ. Appl. 22 (2015), 1089-1114 DOI 10.1007/s00030-015-0315-4

This note corrects the construction of a super-solution in Part II of Section 6 for the case $2 \leq p \leq n$. Please refer to the original article for this part of the discussion and the equation numbers. Let $h$ be as defined in (1.3), $M=\sup _{P_{T}} h$ and $m=\inf _{P_{T}} h$.

We made use of scaling to render the second term on the left side of (6.9) small. However, the condition in (6.13), i.e,

$$
\lambda \log \left(\frac{M^{1 / \lambda}+2 \hat{\varepsilon}}{\hat{h}(y, \hat{s})+2 \hat{\varepsilon}}\right)<1,
$$

for small $\hat{\varepsilon}$, holds if $\log [(M+2 \varepsilon) /(h(y, s)+2 \varepsilon)]<1$. Since $\varepsilon>0$ is small, $M / m \leq e$. In this note, we provide an alternative that removes this restriction.

\section{Super-solutions in Section 6 and Theorem $\mathbf{1 . 2}$}

Part II: Super-solutions for $2 \leq p \leq n$. We construct a function $\eta>0$ such that

$$
\Delta_{p} \eta+(p-1)|D \eta|^{p}-(p-1) \eta_{t} \leq 0, \quad \text { in } \Omega_{T} \quad \text { and } \quad \eta \geq \log h \quad \text { on } \quad P_{T} .
$$

Recall from the paper that $m>0$ and $\varepsilon>0$, small, so that $m-2 \varepsilon>0$.

Let $(y, s) \in P_{T}$ with $s>0$. Then there are $\delta_{0}>0$ and $\tau_{0}>0$ such that $\forall(x, t) \in P_{T}$ with $|x-y| \leq \delta_{0}$ and $|s-t| \leq \tau_{0}$, we have

$$
h(y, s)-\varepsilon \leq h(x, t) \leq h(y, s)+\varepsilon .
$$


Lemma 1.1. Let $A>0, B>0, \beta>0$ and

$$
j(r)=\log \left(A-\frac{B}{r^{\beta}}\right), \quad \text { in } r>(B / A)^{1 / \beta} .
$$

Set $\Gamma=(n-p)-(p-1) \beta$. Then,

$$
\Delta_{p} j+(p-1)|D j|^{p}=\left(\frac{\Gamma}{r^{p}}\right)\left(\frac{\beta B}{A r^{\beta}-B}\right)^{p-1}, \quad \text { in } r>(B / A)^{1 / \beta} .
$$

Proof. We make use of the following expression, i.e., in $r>0$,

$$
\Delta_{p} j+(p-1)|D j|^{p}=\left|j(r)^{\prime}\right|^{p-2}\left((p-1) j(r)^{\prime \prime}+\frac{n-1}{r} j(r)^{\prime}\right)+(p-1)\left|j(r)^{\prime}\right|^{p} .
$$

Differentiating,

$$
\begin{aligned}
j(r)^{\prime} & =\frac{\beta B}{A r^{\beta+1}-B r}, \quad r j(r)^{\prime}=\frac{\beta B}{A r^{\beta}-B} \quad \text { and } \\
j(r)^{\prime \prime} & =-\beta B\left[\frac{(\beta+1) A r^{\beta}-B}{\left(A r^{\beta+1}-B r\right)^{2}}\right] .
\end{aligned}
$$

Using (1.4),

$$
j(r)^{\prime \prime}=-\frac{j(r)^{\prime}}{r}\left(\frac{A(\beta+1) r^{\beta}-B}{A r^{\beta}-B}\right) .
$$

Note that $j(r)^{\prime}>0$ and recall that $\Gamma=(n-p)-(p-1) \beta$. Using (1.3), (1.4) and (1.5)

$$
\begin{aligned}
\Delta_{p} j & =\left(j(r)^{\prime}\right)^{p-2}\left[(n-1) \frac{j(r)^{\prime}}{r}-(p-1) \frac{j(r)^{\prime}}{r}\left(\frac{(\beta+1) A r^{\beta}-B}{A r^{\beta}-B}\right)\right] \\
& =\frac{\left(j(r)^{\prime}\right)^{p-1}}{r}\left[(n-1)-(p-1)\left(\frac{\beta A r^{\beta}+\left(A r^{\beta}-B\right)}{A r^{\beta}-B}\right)\right] \\
& =\frac{\left(j(r)^{\prime}\right)^{p-1}}{r}\left[\frac{(n-p)\left(A r^{\beta}-B\right)-(p-1) \beta A r^{\beta}}{A r^{\beta}-B}\right] \\
& =\frac{\left(j(r)^{\prime}\right)^{p-1}}{r}\left(\frac{\Gamma A r^{\beta}-(n-p) B}{A r^{\beta}-B}\right) .
\end{aligned}
$$

Using (1.3), (1.4) and (1.6) we get

$$
\begin{aligned}
\Delta_{p} j+(p-1)|D j|^{p} & =\frac{\left(j(r)^{\prime}\right)^{p-1}}{r}\left[\frac{\Gamma A r^{\beta}-(n-p) B}{A r^{\beta}-B}+(p-1) r j(r)^{\prime}\right] \\
& =\frac{\left(j(r)^{\prime}\right)^{p-1}}{r}\left[\frac{\Gamma A r^{\beta}-(n-p) B+(p-1) \beta B}{A r^{\beta}-B}\right] \\
& =\frac{\Gamma\left(j(r)^{\prime}\right)^{p-1}}{r}=\frac{\Gamma}{r^{p}}\left(\frac{\beta B}{A r^{\beta}-B}\right)^{p-1} .
\end{aligned}
$$

The lemma follows. 
We construct now a super-solution $\eta$ as follows. Let $\beta, 0<\tau \leq \tau_{0}$, small, $\ell>0, \theta>0, \rho>0$ such that

$$
\begin{aligned}
& \beta=\frac{n-1}{p-1}, \quad \ell=\frac{1}{\tau} \log \left(\frac{M+2 \varepsilon}{h(y, s)+2 \varepsilon}\right), \quad \log \left(\frac{1+\theta-2^{-\beta}}{\theta}\right)=\ell \tau, \quad \text { and } \\
& 0<\rho \leq \min \left[\frac{\delta_{0}}{4}, \frac{1}{2 \ell^{1 / p}}\left(\frac{\beta}{\left[2^{\beta}(\theta+1)-1\right]}\right)^{(p-1) / p}\right]
\end{aligned}
$$

With $\theta$ as in (1.7), set

$$
F(\theta, \rho, r)=\log \left(\frac{1+\theta-(\rho / r)^{\beta}}{\theta}\right), \quad \rho \leq r \leq 2 \rho .
$$

Then $F$ is increasing in $r$,
(i) $F(\theta, \rho, \rho)=0$,
(ii) $F(\theta, \rho, 2 \rho)=\ell \tau$,
(iii) $F \leq 0$, for $r \leq \rho$, and
(iv) $F \geq 0$, for $r \geq \rho$.

Let $(y, s)$ be as in (1.2). By the outer ball condition, there is a $\gamma_{0}>0$ such that for each $0<\gamma \leq \gamma_{0}$, there is a $z \in \mathbb{R}^{n} \backslash \bar{\Omega}$ (depending on $\gamma$ ) with $B_{\gamma}(z) \subset \mathbb{R}^{n} \backslash \bar{\Omega}$ and $y \in \partial B_{\gamma}(z)$.

From hereon, $\tau$ and $\rho$ satisfy (1.7) with the additional imposition that

$$
0<\rho<\gamma_{0} .
$$

Set $r=|x-z|$ and $S_{\rho, \tau}(z)=\left(B_{2 \rho}(z) \backslash B_{\rho}(z)\right) \times[s-\tau, s+\tau]$, the cylindrical shell centered at $(z, s)$. Clearly, $\bar{S}_{\rho, \tau}(z) \subset \bar{B}_{\delta_{0}}(y) \times[s-\tau, s+\tau]$. Let $S^{+}$denote the part of $S_{\rho, \tau}(z)$ in $s \leq t \leq s+\tau$ and let $S^{-}$the part in $s-\tau \leq t \leq s$.

Define

$$
\psi(r, t)= \begin{cases}\ell(s-t)+\ell \tau-F(\theta, \rho, r), & \forall(r, t) \in S^{+} \\ \ell(t-s)+\ell \tau-F(\theta, \rho, r), & \forall(r, t) \in S^{-}\end{cases}
$$

Using (1.8) and (1.9), $\psi$ is $C^{\infty}$ in $x$, in $r>\rho$, and Lipschitz continuous in $t$. Moreover,

$$
\begin{gathered}
\psi(\rho, t)=\ell(s+\tau-t) \quad \text { in } \quad s \leq t \leq s+\tau, \quad \psi(\rho, t)=\ell(t-(s-\tau)) \\
\text { in } \quad s-\tau \leq t \leq s, \quad \text { and } \quad \psi(r, s)=\ell \tau-F(\theta, \rho, r), \quad \text { in } \rho \leq r \leq 2 \rho .
\end{gathered}
$$

Also, $\psi(r, s \pm \tau) \leq 0, r \geq \rho$, implying that $\psi(r, s \pm \tau)=0$ if and only if $r=\rho$.

Next, recalling (1.8), (1.9) and (1.10) set

$$
R=\{(r, t) \in S: \psi(r, t) \geq 0\} .
$$

Call $R^{+}=S^{+} \cap R$ and $R^{-}=S^{-} \cap R$. It is clear that $R \subset \Omega_{T} \cap\left(B_{\delta_{0}}(y) \times\right.$ $[s-\tau, s+\tau])$. Here, $R \cap\{t=s\}=B_{2 \rho}(y) \backslash B_{\rho}(y)$, is the widest section, and $R$ narrows as $|t-s|$ increases to $\tau$. See discussion following (1.10).

In $\bar{\Omega}_{T}$, define the indent function

$$
\eta(x, t)= \begin{cases}\log (M+2 \varepsilon)-\psi(r, t), & \forall(x, t) \in R, \\ \log (M+2 \varepsilon), & \forall(x, t) \in \bar{\Omega}_{T} \backslash R .\end{cases}
$$


Using (1.2), (1.7), (1.8) and (1.10) we see that

(i) $\eta(y, s)=\log (h(y, s)+2 \varepsilon), \quad\left(\right.$ ii) $\eta(x, t) \leq \log (M+2 \varepsilon), \quad \forall(x, t) \in \bar{\Omega}_{T}$,

(iii) $\log h(x, t) \leq \log (h(y, s)+2 \varepsilon) \leq \eta(x, t) \leq \log (M+2 \varepsilon)$,

$\forall(x, t) \in R \cap P_{T}, \quad$ and

(iv) $\eta(x, t) \geq \log h(x, t), \quad \forall(x, t) \in P_{T}$.

If we show that $\eta$ is a super-solution in $\Omega_{T}$, the observations listed above would imply that $\eta$ is a super-solution of (1.1). First we show that $\eta$ is a super-solution in $R \cap \Omega_{T}$. Lemma 5.1 of the original article will then show that $\eta$ is a super-solution in $\Omega_{T}$.

Case $t \neq s$ : Using (1.10) and (1.12), we observe that $\eta_{t}=\ell$ in $R^{+}$and $\eta_{t}=-\ell$ in $R^{-}$. The choice in (1.7) implies that $\Gamma=-(p-1)$. In Lemma 1.1 , take $A=(1+1 / \theta)$ and $B=\rho^{\beta} / \theta$ then (1.7), (1.8) and (1.12) imply in $\rho \leq r \leq 2 \rho$ and $s-\tau \leq t \leq s+\tau$ that

$$
\begin{aligned}
& \Delta_{p} \eta+(p-1)|D \eta|^{p}-(p-1) \eta_{t}=\Delta_{p} F+(p-1)|D F|^{p} \pm(p-1) \ell \\
& \leq(p-1)\left(\ell-\frac{1}{r^{p}}\left(\frac{\beta B}{A r^{\beta}-B}\right)^{p-1}\right) \\
& \quad=(p-1)\left(\ell-\frac{1}{r^{p}}\left(\frac{\beta \rho^{\beta}}{\theta\left[\left(1+\frac{1}{\theta}\right) r^{\beta}-\frac{\rho^{\beta}}{\theta}\right]}\right)^{p-1}\right) \leq 0,
\end{aligned}
$$

by taking $r=2 \rho$ in the above and using (1.7). The case $t=s$ is similar to the proof in Part II of Section 5 in the original article.

Tilak Bhattacharya

Department of Mathematics

Western Kentucky University

Bowling Green

KY 42101

USA

e-mail: tilak.bhattacharya@wku.edu

Leonardo Marazzi

Department of Liberal Arts

Savannah College of Arts and design

Savannah

GA 31401

USA

e-mail: lmarazzi@scad.edu 